\title{
REVIEWS
}

\section{Prevention of Iatrogenic Anemia in Critical and Neonatal Care}

\author{
Department of Hematology, Oncology and Internal Diseases, Medical University of Warsaw, Poland
}

A - research concept and design; $\mathbf{B}$ - collection and/or assembly of data; $\mathbf{C}$ - data analysis and interpretation;

$\mathbf{D}$ - writing the article; $\mathbf{E}$ - critical revision of the article; $\mathbf{F}$ - final approval of article

\begin{abstract}
Iatrogenic anemia caused by diagnostic blood sampling is a common problem in the intensive care unit, where continuous monitoring of blood parameters is very often required. Cumulative blood loss associated with phlebotomy along with other factors render this group of patients particularly susceptible to anemia. As it has been proven that anemia in this group of patients leads to inferior outcomes, packed red blood cell transfusions are used to alleviate possible threats associated with low hemoglobin concentration. However, the use of blood components is a procedure conferring a set of risks to the patients despite improvements in safety. Iatrogenic blood loss has also gained particular attention in neonatal care, where cumulative blood loss due to samples taken during the first week of life could easily equal or exceed circulating blood volume. This review summarizes the current knowledge on the causes of iatrogenic anemia and discusses the most common preventive measures taken to reduce diagnostic blood loss and the requirement for blood component transfusions in the aforementioned clinical situations (Adv Clin Exp Med 2016, 25, 1, 191-197).
\end{abstract}

Key words: intensive care unit, anemia, iatrogenic disease, neonatal intensive care, blood transfusion.

Iatrogenic anemia is an anemia caused by healthcare professionals through diagnostic blood sampling. With advancements in medicine in which there is a constant demand for more laboratory tests to provide state-of-the-art care, the amount of blood drawn can easily reach levels that cause a decrease in hemoglobin concentration to an anemic level. This is particularly true in neonatal and intensive care units, where physicians rely on a continuous monitoring of laboratory values and samples are often taken many times a day. Even if justified, the number of blood tests sometimes exceeds its purpose and can perpetuate anemia in patients who are already medically compromised.

The problem of iatrogenic anemia has been most extensively studied and cared for in the neonatal setting, where literally every drop of blood counts. If one did not have guidelines for diagnostic testing in neonates, one could easily draw enough blood to deplete a patient's entire circulatory blood volume. The response has been to implement various measures which reduce the amount of blood taken for laboratory tests, such as: the use of pediatric test tubes and the introduction of "Point of Care" analyzers [1].

In the intensive care unit, we often observe a great amount of blood sampling in order to receive continuous feedback regarding the status of the patient. The patient becomes an icon and testing replaces the physical exam and interview as the standard of care [2]. There are limited guidelines for how and when to draw blood in adults, which puts patients at greater risks for developing iatrogenic anemia [3]. In contrast, in the neonatal ward, where there is a greater susceptibility of children to iatrogenic anemia, the ways of preventing iatrogenic anemia have been well defined.

Most ICU units have poor methods of regulatory control to reduce the occurrence of iatrogenic anemia. In some situations, such as hematopoietic stem cell transplantation performed in hematologic intensive care units, blood drawn for testing can easily reach $100 \mathrm{~mL}$ per day when complications 
arise $(2 \times$ basic blood morphology, coagulation, biochemistry, cross match, blood cultures -3 sets of double cultures and blood loss due to the volume that has to be discarded from catheters before samples can be taken). Indeed, $100 \mathrm{~mL}$ of blood drawn for diagnostic tests can result in a significant decrease of hematocrit by $1.9 \%$ [4]. As important as blood count monitoring is in the hematological ICU, studies that address the question to what extent iatrogenic anemia may be seen in patients after hematopoietic stem cell transplantation (HSCT) have not been done.

In this short review we will briefly discuss preventive measures against iatrogenic anemia in two of the most common settings where it occurs: the neonatal intensive care unit (NICU) and the ICU. We will look at their advantages, limitations, and how they may be implemented in the care of patients.

\section{Prevention of Iatrogenic Anemia in Pediatric Care}

In the neonatal ward, anemia in premature neonates depends on: the blood volume at birth (due to early or late clamping of the umbilical cord), the rate of erythropoiesis and the amount of blood loss due to blood sampling [1]. According to several studies, diagnostic testing is the main reason of blood loss during ICU stay in all age groups [5-7]. The presence of arterial lines or central venous catheters contributes to a 2.3- to 4-fold higher median number of blood samplings per day [8]. The indications for placing a central venous catheter, such as fluid resuscitation and drug administration, facilitate more efficient medical care; however blood sampling from such lines, including the arterial lines, is extensive since collecting blood is made more convenient and more accessible by an already placed needle. A study performed by Valentine et al., which aimed to determine the amount of blood loss in the pediatric intensive care unit, showed that samples drawn from indwelling central venous catheters have significantly greater overdraw volumes compared to peripheral venous catheters $(143 \% \pm 39 \%, \mathrm{p}<0.001)$ [9].

The number of blood samples drawn by already placed lines can be over-abusive, especially since the blood volume in children is about $80 \mathrm{~mL} / \mathrm{kg}$. A $3 \mathrm{~kg}$ newborn would thus have a circulating volume of about $240 \mathrm{~mL}$. Evidently, this information has been implemented in the methods and protocols for diagnostic testing in the neonatal ward. In a neonate with a weight of less than $1500 \mathrm{~g}, 1 \mathrm{~mL}$ of blood would represent more than $1 \%$ of their total blood volume [5].
One of the measures that most neonatal and other pediatric wards have implemented to reduce blood loss is the use of pediatric-sized tubes, forcing the technician to naturally draw a smaller volume of blood. However, the use of pediatric test tubes faces its own challenges. Most analyzers are designed for standard adult tubes with a blood volume of 5-7 $\mathrm{mL}$ in comparison to a $500 \mu \mathrm{L}$ test-tube used in pediatrics [10]. The laboratory therefore must prepare to have an analyzer to handle the smaller pediatric tubes, because the lack of proper equipment will result in manual processing, which may lead to human errors during different phases of blood analysis [10].

In the last decade we have seen the introduction of Point of Care (POC) analyzers for diagnostic testing. This newer method prevents unnecessary blood loss from blood sampling. It is an instrument that allows tests to be performed outside of the hospital laboratory setting, and most importantly, it only requires a small volume of blood, which has the potential to reduce iatrogenic anemia $[10,11]$. The POC analyzer has earned special favor in the ICU because it reduces the time needed for the traditional steps in laboratory processing such as specimen transport, preparation of the specimen and data entry. It allows physicians to almost instantly receive pertinent information about a patient's status. This is crucial in situations where the status of patients continuously changes. By using quicker measures to obtain diagnostic information about a patient, physicians should be able to respond better clinically [12]. The fact that the POC only requires a small volume of blood in comparison to traditional phlebotomy practices, allows a decrease in blood loss and in turn, a decreased need for blood products, which is particularly important for infants, neonates and fragile patients that one may find in the ICU [12]. An example of the use of POC in the adult ICU is when measuring cardiac markers in a patient with suspected myocardial infarction. The POC analyzer will provide the results of the blood test in minutes and the patient may be quickly sent for percutaneous coronary intervention (PCI).

Several studies have been performed to compare the values of different hematological parameters obtained with the standard methodology of phlebotomy to those of the POC analyzers' microsystem. Their aim was to validate the introduction of the microsystem into everyday clinical practice. In a study conducted by Papa et al., capillary and venous blood samples were taken from 150 neonates. All blood samples were processed in their traditional blood analyzer, Sysmex XE-2100 (TOA Medical Electronics, Kobe, Japan), and output a complete blood count and differential counts 
of leukocytes, reticulocytes and erythroblasts. Additionally, 10 microliters from each sample were analyzed using the POC instrument, ABXMicros-CRP200 (Horiba Medical, Montpellier, France). The POC analyzer calculated the following parameters within $70 \mathrm{~s}$ : white blood cell (WBC) count, red blood cell (RBC) count, hemoglobin ( $\mathrm{Hb})$ concentration, hematocrit (Hct), mean corpuscular volume (MCV), mean corpuscular hemoglobin $(\mathrm{MCH})$, mean corpuscular hemoglobin concentration (MCHC), red cell distribution width (RDW), platelet count (PLT), plateletcrit (PCT), mean platelet volume (MPV), platelet distribution width (PDW), and the percentages and absolute counts of lymphocytes, monocytes and granulocytes [1]. Their results indicated that the data obtained from the ABXmicros was reliable enough for the measurement of $\mathrm{Hb}$ concentration but the $\mathrm{RBC}$ and platelet count needed to be read with caution because of a slight overestimation of these values. The advantage is nevertheless that POC testing utilizes approximately $18 \mu \mathrm{L}$ of blood per testing, whereas the standard pediatric test tube uses $3.5 \mathrm{~mL}$ (adults $8.5 \mathrm{~mL}$ ) [13]. Moreover, the immediate availability of results allows the possibility of rapid medical intervention [1], which not only is vital to the care of critical neonates but also in the adult ICU, as it may optimize transfusion requirements [14].

The use of POC has the possibility of reducing the number of RBC transfusions. In a study at Stanford Medical University, it was noted that low birth weight infants experience extensive blood sampling, which results in anemia and consequently RBC transfusions. When clinicians introduced a POC system called iSTAT, they noticed a statistical difference. The iSTAT measures $\mathrm{pH}, \mathrm{pCO} 2$, hemoglobin, hematocrit, $\mathrm{Na}+\mathrm{K}+$ and $\mathrm{Ca} 2+$. The implementation of this device resulted in a $30 \%$ decrease in total number of phlebotomies and a $43 \%$ decrease in transfusions in a group of 2-week-old neonates [15].

The POC system has gained popularity in many fields and this has led to a wide range of analyzers developed to measure different values of markers, electrolytes and other substances in the blood, and even more are being developed as the trend in countries like the USA shows an increase in the use of bedside point of care testing (POCT) [16]. As the usage of the POC analyzers becomes more common, regulatory standards have been critically imposed.

Spectroscopic noninvasive measurement might be considered as an alternative to blood sampling [17]. It is described as a modality that will both combat iatrogenic anemia and painful blood sampling simultaneously. This device uses a hand-held probe on the forearm with a halogen white light which passes through skin. The change in light wavelength is compared to a standard control to obtain a value of hemoglobin concentration. It was the first report of a noninvasive estimation of hemoglobin from the skin of pre-term infants with good correlation to venous hemoglobin values, thus offering an alternative noninvasive method of measuring hemoglobin level. However the function of this device is limited to the measure of hemoglobin and more data is often needed in the intensive care unit. It shows that there is potential for alternative ways of dealing with the problem of iatrogenic anemia [18].

\section{Prevention of Iatrogenic Anemia in Intensive Care Units}

The ICU is a setting with a high rate of physician consultations but is usually not governed by blood sampling guidelines. Every new physician introduced into a patient's management of care may decide to order their own lab results. This fact is often overlooked, but if the ICU would formulate their own guidelines for blood testing, independent to the wishes of consulting physicians, the number of blood test could in some cases be reduced 20-fold [19].

The physician's decision to draw blood for tests can be regulated by special protocols [20]. A computerized support system tracks lab, pharmacology, radiology and other services ordered by physicians, allowing them to benchmark the performance of various physicians. This was done by comparing the activity profiles of several physicians, and in doing so, they managed to reduce the orders of CBC's and basic metabolic profiles by $25-30 \%$ [21]. This reduction is substantial and decreases blood loss and resulting iatrogenic anemia. Tracking physicians' work may seem intrusive and in some ways offensive, however, it also a helpful way of optimizing a physician's work, which is beneficial for patient care.

At a surgical intensive care unit in Geneva, Switzerland, guidelines were created based on regular feedback from appropriate tests for arterial blood gases (ABGs). Arterial blood gases is the most common lab test done in the ICU and indications for this test are often up to the physician's own judgment [22]. A physician is meant to know when to order the appropriate laboratory tests, but as it often may get stressful during working-shifts, observations like respiratory rate or altered pattern of breathing were seldom taken into account in the decision to measure ABGs. Subsequently, 
an algorithm was proposed that took into account new findings in patient status as well as cutaneous oxygen saturation into standard information of the partial pressure of carbon dioxide. The algorithm would then inform the physician at what time intervals it would be necessary to measure blood gases again. However, the benefit of using the algorithm was meager and, in general, did not influence patients' statuses or surgical outcomes. The average number of blood gas analyses dropped from 8.2 to 4.8 per patient day. The length of stay and mortality rate was the same as in the control group [22]. The report, however, did not look at the effect on hemoglobin, which would require another study.

Anemia of chronic disease is a well-elicited condition, where patients have a blunted erythropoietin response and cannot mobilize iron storage for hemoglobin production. Critically ill patients also have, in addition to the many reasons that can cause anemia in the ICU (overt or occult blood loss routes, red blood cell senescence, hemodilution), including our main focus on frequent phlebotomies, a lower erythropoietin response [23]. There is a natural increase in the response of erythropoietin as anemia progresses, which is a normal physiological feedback loop, but it does not always occur in critically ill patients. Exacerbating blood-loss will thus have a greater impact on ICU patients who are not fit to recover from phlebotomy compared to an otherwise-healthy person. Thus, such patients often require $\mathrm{PRBC}$ transfusions to alleviate the possible risks associated with anemia. A few randomized trials aimed at guiding transfusion policy in intensive care units have been performed, but for now, there is no clear conclusion speaking in favor of a liberal transfusion strategy (transfusion trigger $=10 \mathrm{~g} / \mathrm{dL}, \mathrm{Hb}$ target $=10-12 \mathrm{~g} / \mathrm{dL}$ ) [24]. It seems that very ill patients without cardiovascular comorbidities might benefit from more restrictive transfusion strategies (transfusion trigger $=7 \mathrm{~g} / \mathrm{dL}, \mathrm{Hb}$ target $=7-9 \mathrm{~g} / \mathrm{dL}$ ) [25]. Therefore, $\mathrm{BJH}$ recommends a default transfusion threshold of $7 \mathrm{~g} / \mathrm{dL}$ and a target of 7-9 g/dL in most critically ill patients [24]. Interestingly, a new international randomized trial involving patients with septic shock in the ICU showed no significant differences in mortality between groups of patients who underwent PRBC transfusions with lower and higher hemoglobin thresholds [26].

Administration of epoetin alfa in critically ill patients leads to a significant decrease in packed $\mathrm{RBC}$ transfusion requirements in patients receiving epoetin alfa $[27,28]$. Although this type of prophylaxis for critically ill patients may not always be cost-efficient, even though it may reduce the need for blood transfusions, it is definitely worth looking into as a way of preventing iatrogenic anemia and requires further study. At present, the official guidelines published by the British Journal of Haematology clearly state that "erythropoietin should not be used to treat anaemia in critically ill patients until further safety and efficacy data are available" [24].

Patients suffering from chronic kidney disease are also susceptible to anemia because of erythropoietin underproduction associated with renal failure. Erythropoiesis stimulating agents (ESA) are widely used in clinical practice to augment hemoglobin level in this group of patients. ESA prevent further advancement of renal dysfunction along with the development of cardiovascular disease [29]. However, inflammatory states, which often occur in critical care patients, have been recognized as a factor which may diminish the response to erythropoiesis stimulating agents. [30] Thus, it seems plausible that this group of patients could particularly benefit from measures taken to reduce diagnostic blood loss.

Lyon et al. suggested a mathematical model that can be used to anticipate hemoglobin level decrement in patients who are exposed to repetitive diagnostic blood loss due to blood sampling [31]. The model takes into account two factors contributing to hemoglobin loss: red blood cell senescence and diagnostic blood loss due to phlebotomy. It can be easily adjusted to predict the onset of anemia in critical care patients by setting the hemoglobin synthesis rate to zero, based on the fact that bone marrow suppression and insufficient erythropoiesis often occur in these patients. The model shows that most female and male patients will reach the transfusion threshold of $\mathrm{Hb} 7 \mathrm{~g} / \mathrm{dL}$ within 30 and 24 days, respectively.

The use of pediatric test tubes in the adult setting is another way of reducing blood loss. The actual blood required to measure data is $3-100 \mu \mathrm{L}$, but it is common practice that phlebotomists draw full large-volume tubes regardless [32]. SanchezGiron et al. demonstrated that by using small-volume tubes (SVT), the overall median blood-loss from laboratory testing (BLLT) could be reduced by $73 \%$ and $74 \%$ in critical patients, from an original median BLLT of $159.8 \mathrm{~mL}$ to $61.8 \mathrm{~mL}$. The study pointed out that the most frequently ordered tests were in chemistry, hematology, and coagulation, which were done in $1.1,1.2$, and $1.5 \mathrm{~mL}$ tubes, respectively. The $2.7 \mathrm{~mL}$ tubes were rarely encountered and was noted for use in only one situation for each testing category (e.g. chemistry: total iron binding capacity). The main concern with the use of SVT was that many laboratories lack equipment for analyzing them (as discussed previously). There are certain laboratory instruments needed to handle the small volume tubes, and if not available, some of the diagnostic steps need to 
be done manually. This is not only time consuming but may also cause errors. Though it is a beneficial technique, at this time, the use of SVT can only be reserved for those laboratories capable of handling them.

Another blood conserving method is to try to reduce the volume of discarded blood taken before obtaining a specimen [33]. The first batch of filling blood, e.g. from the central line, has to be discarded as it might have different blood values than circulating blood. The volume discarded can easily range from $18-30 \%$ of the total blood drawn, and has been said to be one of the contributors to the extensive blood loss seen in iatrogenic anemia. MacIsaac et al. conducted a study using a VAMP (venous arterial blood management protection) plus system, which allows the phlebotomist to withdraw the "blank" blood using the syringe attached to the needle and then re-infuse the "blank" blood after obtaining the appropriate blood specimen. The study focused on confirming or rejecting the theory that the "blank" played a role in the decrease of hemoglobin in ICU patients. Unfortunately, their results demonstrated that only a minuscule (20 mL/day) amount was saved by this method and that there was only a small increase in hemoglobin level in a minority of the non-transfused patients. However, in that particular minority, the researchers did not control the fluid resuscitation volume, and they concluded that the rise in hemoglobin probably was attributed to this uncontrolled variable and not the saving of the "blank". [33]

\section{Summary}

There are many factors that contribute to iatrogenic anemia in different specialties of medicine. Laboratory testing drives a large percentage of medical decision making. So far, the main factor which controls unnecessarily frequent testing is the increase in hospital financial costs, where the overuse of blood sampling, together with other diagnostic tests, are major expenses [3]. What happens when there is no roof to how much money can be spent on laboratory tests? This may only apply to some healthcare systems, and perhaps in such a case, a tight budget works beneficially in the fight against iatrogenic anemia. The lack of guidelines in phlebotomy practice is a sensitive subject because it is up to the physician how often they want to order blood tests. Even though most physicians know that there is no reason to draw blood every $20 \mathrm{~min}$, in many hospitals there is nothing that limits them to do so unless there is a formal policy. Many times young and inexperienced physicians are frightened to miss a change in their patient's status and thus want to exclude every possibility, even though most of the tests will not add anything to the clinical picture. Would guidelines detailing how to order laboratory test address this issue? And would this take away the independence of the physician or simply optimize the physician's work?

Most of the methods used for optimization mentioned in this review rely on reducing the use of blood sampling, which not only has an impact on blood loss, but is economical; and that is in every hospital's interest. Improvements in this area would also reduce unnecessary workload for physicians, as each test drawn has to be later assessed and recorded. The use of POC analyzers allows routing information to be available faster and more efficiently. Then the physician can ask the question as to whether their patient would benefit from more testing. According to a report from a collaboration of different specialties in Italy, POC results were sufficient for measuring hemoglobin concentration, but the RBC and platelet counts needed to be read with caution [1]. When the POC analyzer first began to be used, the data was manually recorded. This has changed to become automated to reduce work and assure quality. However, even with the advances in device design, most POC analyzers do not have error prevention features. In such a case, a Point-of-Care inspection list as formulated by Lewandrowski, is invaluable in assuring quality test results.

The simple solutions of changing to less arbitrary testing, and using smaller volumes should not only lead to savings but also to a reduction in iatrogenic anemia for the hospitals which acknowledge this problem.

\section{References}

[1] Papa F, Rongioletti M, Ventura MD, Di Turi F, Cortesi M, Pasqualetti P, Majolini MB, Collegiani V, Cicchese M, Notarmuzi ML, Agostino R, Liumbruno GM: Blood cell counting in neonates: a comparison between a low volume micromethod and the standard laboratory method. Blood Transfus 2011, 9, 400-406.

[2] Verghese A: Culture shock - patient as icon, icon as patient. N Engl J Med 2008, 25, 2748-2751.

[3] Woodhouse S: Complication of critical care: lab testing and iatrogenic anemia. MLO Med Lab Obs 2001, 33, 28-31.

[4] Tavendiranathan P, Bagai A, Ebidia A, Detsky AS, Choudhry NK: Do blood tests cause anemia in hospitalized patients? The effect of diagnostic phlebotomy on hemoglobin and hematocrit levels. J Gen Intern Med 2005, 20, 520-524. 
[5] Nexø E, Christensen NC, Olesen H: Volume of blood removed for analytical purposes during hospitalization of low-birthweight infants. Clin Chem 1981, 27, 759-761.

[6] Bell EF: When to transfuse preterm babies. Arc Dis Child Fetal Neonatal Ed 2008, 93, 469-473.

[7] Widness JA: Treatment and prevention of neonatal anaemia. Neoreviews 2008, 9, 526-533.

[8] Bateman ST, Lacroix J, Boven K, Forbes P, Barton R, Thomas NJ, Jacobs B, Markovitz B, Goldstein B, Hanson JH, Li HA, Randolph AG: Pediatric Acute Lung Injury and Sepsis Investigators Network Anemia, blood loss, and blood transfusions in North American children in the intensive care unit. Am J Respir Crit Care Med 2008, 178, 26-33.

[9] Valentine SL, Bateman ST: Identifying factors to minimize phlebotomy-induced blood loss in the pediatric intensive care unit. Pediatr Crit Care Med 2012, 13, 22-27.

[10] Demir AY, van Solinge WW, Kemperman H: Handling of and direct sampling from primary Barcode-labeled pediatric tubes on Vitros Clinical Chemistry analyzers integrated into an enGen Work Cell. Clin Chem 2005, 51, 920-921.

[11] Briggs C, Guthrie D, Hyde K, Mackie I, Parker N, Popek M, Porter N, Stephens C: British Committee for Standards in Haematology General Haematology Task Force: Guidelines for point-of-care testing: haematology. Br J Haematol 2008, 142, 904-915.

[12] Briggs C, Carter J, Lee SH, Sandhaus L, Simon-Lopez R, Vives Corrons JL: International Council for Standardization in Haematology: ICSH Guideline for worldwide point-of-care testing in haematology with special reference to the complete blood count. Int J Lab Hematol 2008, 30, 105-116.

[13] St. Louis P: Status of point-of-care testing: promise, realities, and possibilities. Clin Biochem 2000, 33, 427-440.

[14] Hitt E: Smaller blood tubes reduce the amount of blood drawn for lab test without affecting results. March 2011: http://www.medscape.com/viewarticle/718472, referred on $8^{\text {th }}$ April 2012.

[15] Madan A, Kumar R, Adams MM, Benitz WE, Geaghan SM, Widness JA: Reduction in red blood cell transfusions using a bedside analyzer in extremely low birth weight infants. J Perinatol 2005, 25, 21-25.

[16] Scalise D: Poised for growth. Point-of-care testing. Hosp Health Netw 2006, 77-83.

[17] Rabe H, Stupp N, Ozgün M, Harms E, Jungmann H: Measurement of transcutaneous hemoglobin concentration by noninvasive white-light spectroscopy in infants. Pediatrics 2005, 116, 841-843.

[18] Rabe H, Alvarez RF, Whitfield T, Lawson F, Jungmann H: Spectroscopic noninvasive measurement of hemoglobin compared with capillary and venous values in neonates. Neonatology 2010, 98, 1-5.

[19] Corwin HL, Gettinger A, Pearl RG, Fink MP, Levy MM, Abraham E, MacIntyre NR, Shabot MM, Duh MS, Shapiro MJ: The CRIT Study: Anemia and blood transfusion in the critically ill - current clinical practice in the United States. Crit Care Med 2004, 32, 39-52.

[20] Pohlman AS, Carven JH, Lindsay K: Conserving blood in the intensive care unit. Crit Care Nurse 2001, Suppl 1-14, quiz 16.

[21] Studnicki J, Bradham DD, Marshburn J, Foulis PR, Straumfjord JV: A feedback system for reducing excessive laboratory tests. Arch Pathol Lab Med 1993, 117, 35-39.

[22] Merlani P, Garnerin P, Diby M, Ferring M, Ricou B: Quality improvement report: Linking guideline to regular feedback to increase appropriate requests for clinical tests: blood gas analysis in intensive care. BMJ 2001, 323, 620-624.

[23] Rogiers P, Zhang H, Leeman M, Nagler J, Neels H, Mélot C, Vincent JL: Erythropoietin response is blunted in critically ill patients. Intensive Care Med 1997, 23, 159-162.

[24] Retter A, Wyncoll D, Pearse R, Carson D, McKechnie S, Stanworth S, Allard S, Thomas D, Walsh T: British Committee for Standards in Haematology: Guidelines on the management of anaemia and red cell transfusion in adult critically ill patients. Br J Haematol 2013, 160, 445-464.

[25] Hébert PC, Wells G, Blajchman MA, Marshall J, Martin C, Pagliarello G, Tweeddale M, Schweitzer I, Yetisir E: A multicenter, randomized, controlled clinical trial of transfusion requirements in critical care. Transfusion Requirements in Critical Care Investigators, Canadian Critical Care Trials Group. N Engl J Med 1999, 340, 409-417.

[26] Holst LB, Haase N, Wetterslev J, Wernerman J, Guttormsen AB, Karlsson S, Johansson PI, Aneman A, Vang ML, Winding R, Nebrich L, Nibro HL, Rasmussen BS, Lauridsen JR, Nielsen JS, Oldner A, Pettilä V, Cronhjort MB, Andersen LH, Pedersen UG, Reiter N, Wiis J, White JO, Russell L, Thornberg KJ, Hjortrup PB, Müller RG, Møller MH, Steensen M, Tjäder I, Kilsand K, Odeberg-Wernerman S, Sjøbø B, Bundgaard H, Thyø MA, Lodahl D, Mærkedahl R, Albeck C, Illum D, Kruse M, Winkel P, Perner A, TRISS Trial Group: Scandinavian Critical Care Trials Group: Lower vs. higher hemoglobin threshold for transfusion in septic shock. N Engl J Med 2014, 371, 1381-1391.

[27] van Iperen CE, Gaillard CA, Kraaijenhagen RJ, Braam BG, Marx JJ, van de Wiel A: Response of erythropoiesis and iron metabolism to recombinant human erythropoietin in intensive care unit patients. Crit Care Med 2000, $28,2773-2778$.

[28] Corwin HL, Gettinger A, Rodriguez RM, Pearl RG, Gubler KD, Enny C, Colton T, Corwin MJ: Efficacy of recombinant human erythropoietin in the critically ill patient: a randomized, double-blind, placebo-controlled trial. Crit Care Med 1999, 27, 2346-2350.

[29] Kuriyama S, Tomonari H, Yoshida H, Hashimoto T, Kawaguchi Y, Sakai O: Reversal of anemia by erythropoietin therapy retards the progression of chronic renal failure, especially in nondiabetic patients. Nephron 1997, 77, $176-185$. 
[30] Locatelli F, Aljama P, Bárány P, Canaud B, Carrera F, Eckardt KU, Hörl WH, Macdougal IC, Macleod A, Wiecek A, Cameron S: European Best Practice Guidelines Working Group: Revised European best practice guidelines for the management of anaemia in patients with chronic renal failure. Nephrol Dial Transplant 2004, 19, Suppl 2, 1-47.

[31] Lyon AW, Chin AC, Slotsve GA, Lyon ME: Simulation of repetitive diagnostic blood loss and onset of iatrogenic anemia in critical care patients with a mathematical model. Comput Biol Med 2013, 43, 84-90.

[32] Sanchez-Giron F, Alvarez-Mora F: Reduction of blood-loss from laboratory testing in hospitalized adult patients using small-volume (pediatric) tubes. Arch Pathol Lab Med 2008, 132, 1916-1919.

[33] MacIsaac CM, Presneill JJ, Boyce CA, Byron KL, Cade JF: The influence of a blood conserving device on anaemia in intensive care patients. Anaesth Intensive Care 2003, 31, 653-657.

\section{Address for correspondence:}

Natalia Jakacka

Department of Hematology, Oncology and Internal Diseases

Medical University of Warsaw

Banacha 1a

02-097 Warszawa

Poland

E-mail: njakacka@gmail.com

Conflict of interest: None declared

Received: 5.09 .2014

Revised: 26.09.2014

Accepted: 29.10.2014 